\title{
Direct Measurement of the Oscillation Frequency in an Optical-Tweezers Trap by Parametric Excitation
}

\author{
Joji Joykutty, Vaibhav Mathur, V. Venkataraman, and Vasant Natarajan* \\ Department of Physics, Indian Institute of Science, Bangalore 560 012, India
}

(Received 7 February 2005; published 1 November 2005)

\begin{abstract}
We demonstrate a novel technique for direct measurement of the oscillation frequency in an opticaltweezers trap. The technique uses the phenomenon of parametric resonance in an oscillator when the stiffness of the trapping potential is modulated. The trapped particle is a strongly damped oscillator; hence, the signature of parametric resonance is not an increase in the amplitude but an increase in the size of Brownian fluctuations. The trap frequency is measured with an accuracy of $0.1 \%$, which is better than previous techniques and thus opens up new possibilities in experiments with optical tweezers.
\end{abstract}

PACS numbers: 87.80.Cc, 07.60.- j

Optical tweezers $[1,2]$ have become a standard technique for the manipulation of microscopic particles. The technique uses a laser beam that is focused to a near diffraction-limited spot, usually through a high numerical aperture (N.A.) microscope objective. Micron-sized objects (having the correct refractive index) are then trapped at the intensity maximum of the focused laser beam. The technique finds diverse applications in fields ranging from fundamental experiments in physics [3] and biology [4-7] to nanotechnology and nanofabrication. For example, the elastic properties of a single DNA molecule can be studied by attaching it to a polystyrene bead and using optical tweezers to manipulate the bead [8]. Similarly, molecular forces in single motor-protein molecules (such as kinesin) are studied by attaching them to polystyrene beads $[9,10]$. However, to use this technique in such quantitative studies, it is necessary to know the strength of the tweezers trap very precisely. While it is possible to calculate the trap strength theoretically, in practice it depends on several unknown parameters such as the optical alignment into the microscope, absorption along the optical path, and aberrations in the lenses. It is therefore desirable to have a direct and precise calibration of the trap strength.

In this paper, we present a simple technique that allows a direct measurement of the trap-oscillation frequency. The technique uses the phenomenon of parametric resonance in a harmonic oscillator. The focused laser beam produces a harmonic potential for the trapped particle whose strength is proportional to the laser power. The trap strength can thus be modulated by modulating the laser power, which excites the motion of the particle when the modulation frequency matches the resonance condition. Other techniques of measuring the trap strength are generally less accurate. In one commonly used technique [7], the sample is moved relative to the laser beam at a constant speed. The trapped object then gets displaced from the trap center due to the viscous drag of the surrounding medium. However, measuring the force this way requires a precise knowledge of the coefficient of viscosity. Similarly, the trap strength can be determined from the rolloff frequency of the power spectrum of Brownian fluctuations of the trapped particle [10]. Again, this depends on knowledge of the viscous drag coefficient. Alternately, the size of the fluctuations at a given temperature can be directly related to the trap strength using the equipartition theorem. This works well for weak traps, but in strong traps it is susceptible to errors due to technical noise, which adds to the measured fluctuations. Hence, even with careful experimentation, such methods are limited in accuracy to a few percent. By contrast, our technique measures the oscillation frequency directly with $0.1 \%$ accuracy.

Let us first consider the phenomenon of parametric resonance in a one-dimensional oscillator. The equation of motion for a parametrically driven oscillator is

$$
\ddot{x}+\omega_{0}^{2}(1+h \cos \gamma t) x=0,
$$

where $\omega_{0}$ is the natural frequency, $h$ is the depth of modulation $(h \ll 1)$, and $\gamma$ is the parametric drive frequency. The solution to the above equation can be found in standard textbooks on mechanics [11]. In general, parametric resonance is strongest if the drive frequency is close to $2 \omega_{0}$. Hence, we put $\gamma=2 \omega_{0}+\epsilon$, where $\epsilon \ll \omega_{0}$. The (approximate) solution to Eq. (1) may be sought in the form

$$
x=a(t) \cos \left(\omega_{0}+\frac{1}{2} \epsilon\right) t+b(t) \sin \left(\omega_{0}+\frac{1}{2} \epsilon\right) t,
$$

where $a(t)$ and $b(t)$ are slowly varying functions of time compared to the trigonometric functions. Retaining only terms of first order in $\epsilon$, one gets two linear differential equations for the functions $a(t)$ and $b(t)$. The solutions are proportional to $\exp (s t)$ with the condition

$$
s^{2}=\frac{1}{4}\left[\left(\frac{1}{2} h \omega_{0}\right)^{2}-\epsilon^{2}\right] .
$$

The condition for parametric resonance is that $s$ is real; hence, it occurs in the range

$$
-\frac{1}{2} h \omega_{0}<\epsilon<\frac{1}{2} h \omega_{0} .
$$

Thus, the amplitude increases exponentially with time in a 
narrow range around $2 \omega_{0}$. When $\gamma$ is exactly equal to $2 \omega_{0}$, the amplitude increases as $\exp \left(\frac{1}{4} h \omega_{0} t\right)$.

Thus, we see that we can increase the amplitude of motion by giving a parametric drive at the right frequency. Indeed, this is exactly what every child learns intuitively on a swing: The amplitude of the swing can be increased by varying her center of gravity (and, hence, the length of the swing) up and down at twice the oscillation frequency. For our case of a particle in an optical-tweezers trap, the trapping potential (or the trap stiffness) is directly proportional to the laser power. Therefore, parametric driving means that we just have to modulate the laser power sinusoidally. Such parametric amplification can have important applications. For example, if the phase of the parametric drive is chosen correctly, one quadrature component of the oscillation gets attenuated at the cost of an increase in the other. This can be used for so-called "squeezing" of the thermal noise in a classical oscillator [12] for precision measurements, analogous to squeezing of quantum noise by parametric down-conversion of photons.

The above analysis ignores an important aspect of a real system, namely, the presence of friction. This would appear as a term $\lambda \dot{x}$ on the left-hand side of Eq. (1). It is well known that this term causes the amplitude to decay as $\exp (-\lambda t)$. Therefore, in the presence of both parametric driving and friction, the amplitude will vary as $\exp [(s-$ $\lambda) t]$. When $\lambda / \omega_{0}$ is very small, i.e., for an underdamped system, the phenomenon of parametric amplification continues to happen in the presence of damping but occurs over a narrower range because of the $\lambda$ term in the exponential [13]. The situation in the case of an overdamped system (where $\lambda / \omega_{0}$ is very large) is generally not considered important because the damping due to friction will always dominate any parametric amplification. This is indeed the situation for optical trapping of beads and particles: They are generally in a region of low Reynolds number flow and strongly damped. Estimating the value of $\lambda$ from Stokes' law, we find that $\lambda / \omega_{0}$ is about 50 for a trapped polystyrene bead in water.

The fact that the system is strongly damped is probably why parametric excitation has not been studied in optical tweezers before. But there is another important manifestation of this damping: thermal fluctuations or Brownian motion. The fluctuation-dissipation theorem [14] tells us that any system with damping will eventually attain equilibrium and show fluctuations, and the size of the fluctuations depends inversely on the damping. We have seen earlier that, in the presence of parametric excitation, the decay term for an overdamped system reduces from $\exp (-\lambda t)$ to $\exp [-(\lambda-s) t]$. Therefore, the effective damping reduces near the parametric resonance and, consequently, the size of the fluctuations should increase. In analogy with an electrical $L C R$ circuit, the parametric drive injects a negative $R$ into the circuit, but this $R$ does not have any Johnson noise associated with it and, therefore, causes an overall increase in the noise voltage.
Our experimental approach is now clear; we have to look for an increase in the Brownian fluctuations of the trapped particle in the presence of a parametric drive near the $2 \omega_{0}$ resonance. As mentioned earlier, it is easy to modulate the trap stiffness by modulating the laser power. We have modulated the power by placing an acousto-optic modulator (AOM) at the output of the laser. The AOM has a center frequency of $200 \mathrm{MHz}$ and a peak diffraction efficiency of $75 \%$. However, we use the zeroth-order (unshifted) beam for the experiment. This has several advantages: The beam direction remains unshifted so that the optical alignment is not affected; the spatial profile of the beam is unchanged when it passes through the AOM so that it remains pure Gaussian; and we can use almost all the available laser power since we are looking for only a small depth of modulation. The power is modulated by modulating the rf power exciting the crystal. The rf power determines the laser power transferred into the diffracted spots; therefore, varying the rf power also varies the power remaining in the zeroth-order beam. The modulation of the $\mathrm{rf}$ power is adjusted so that the laser power varies by $\pm 5 \%$.

The schematic of the experiment is shown in Fig. 1. The trapping beam is obtained from a diode-pumped Nd:YAG laser with a maximum output power of $400 \mathrm{~mW}$. The output of the laser first passes through the AOM. The beam size is then adjusted using a lens so that it fills the back aperture of the microscope objective. The microscope is a Zeiss inverted microscope with a $100 \times, 1.44$ N.A. oilimmersion objective. The output from a $5 \mathrm{~mW}$ HeNe laser beam is mixed with the primary trapping beam and is used for detecting the trapped particle. The backscattered light from the HeNe laser is detected by a quadrant photodetector (QPD). The output of the QPD is amplified and analyzed to determine the position of the particle. The sample is mounted on a precision $x-y$ translation stage that uses a piezoelectric transducer to position the sample with $1 \mathrm{~nm}$ accuracy. The sample consists of a dilute suspension of $3 \mu \mathrm{m}$ polystyrene beads in an aqueous solution. The solu-

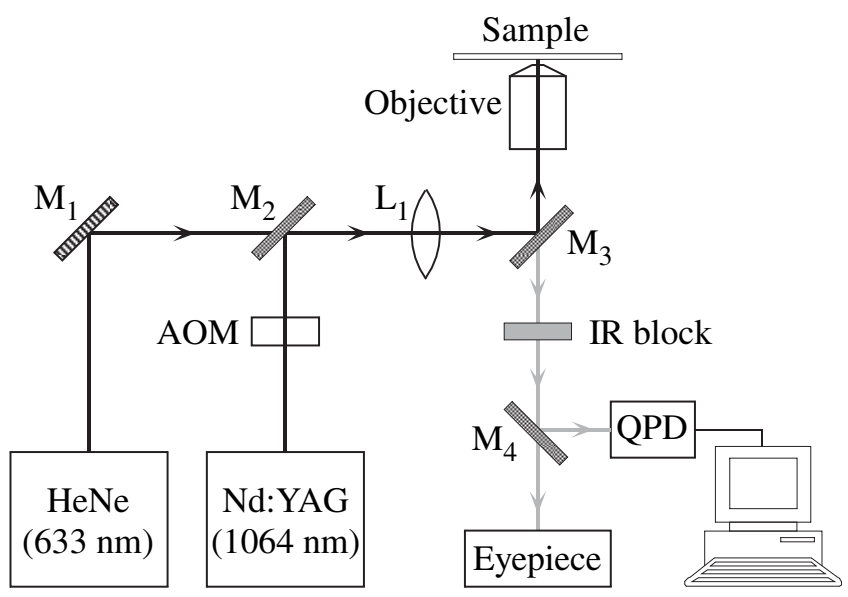

FIG. 1. Schematic of the experiment. The sample is mounted on a precision $x-y$ translation stage. M, mirror; AOM, acoustooptic modulator; L, lens; QPD, quadrant photodetector. 
tion is placed on a $0.15 \mathrm{~mm}$ thick glass slide and held inside an $\mathrm{O}$ ring.

As mentioned before, the experiments were done with $5 \%$ modulation $[h=0.05$ in Eq. (1)] of the laser power using the AOM. For a laser power of $100 \mathrm{~mW}$ (at the specimen plane), the trap strength is about $10^{-5} \mathrm{~N} / \mathrm{m}$, which gives an oscillation frequency of a few $\mathrm{kHz}$ for a $3 \mu \mathrm{m}$ bead. Therefore, the modulation frequency was varied in the range of 3 to $12 \mathrm{kHz}$. The $x$ position of the trapped particle was measured at each frequency for a total of $11 \mathrm{~s}$ (180000 points). The measured variance in the position as a function of modulation frequency is shown in Fig. 2, for an input laser power of $300 \mathrm{~mW}$. The voltage fluctuation seen by the QPD is plotted without scaling to a length since we are interested only in relative changes in this value.

The graph shows a clear parametric resonance near a modulation frequency of $8 \mathrm{kHz}$. The solid curve in the figure is a Lorentzian fit to the measured values, yielding a center frequency of $7.95 \pm 0.01 \mathrm{kHz}$ and a width of $0.1 \mathrm{kHz}$. From Eq. (4), we expect a width of 5\% around $2 \omega_{0}$ where the parametric excitation occurs. But note that this is the total range over which $s$ is real. Since we are looking at a region where this term makes a significant difference to the damping, it seems reasonable that the observed width is only $1 \%$. It is also important to note that there is no significant excitation of the oscillation in this overdamped system. Such an oscillation would show up as a sharp peak at the trap frequency when we take the Fourier transform of the data recorded by the QPD, but we do not observe any such peak.

We have further studied the trap frequency at various laser powers to verify that it shows the expected behavior. The trap stiffness $k_{\text {trap }}$ is directly proportional to the laser power and is related to the trap frequency as $k_{\text {trap }}=$ $4 \pi^{2} f_{\text {trap }}^{2} m$, where $m$ is the mass of the bead. A plot of

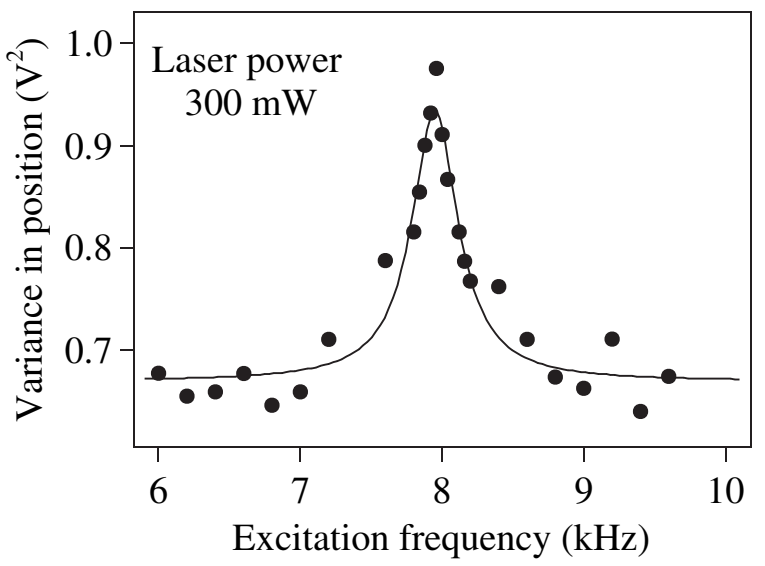

FIG. 2. Parametric amplification. The variance in position along the $x$ direction (given by the voltage fluctuation seen by the QPD) is plotted as a function of the parametric excitation frequency. The solid curve is a Lorentzian fit yielding a center frequency of $7.95 \pm 0.01 \mathrm{kHz}$ and a width of $0.1 \mathrm{kHz}$. $k_{\text {trap }}$ for powers ranging from 225 to $300 \mathrm{~mW}$ is shown in Fig. 3, taking the bead mass to be $1.5 \times 10^{-14} \mathrm{~kg}$. The data shows the expected linear behavior; however, the linear fit gives a threshold power of $130 \mathrm{~mW}$. This means that, if we input $300 \mathrm{~mW}$, only $170 \mathrm{~mW}$ is available for trapping. Note that we have measured only the input power going into the system and not the trapping power at the sample location. Direct measurement of the power at the sample location (after the laser beam passes through an oil-immersion objective and the glass slide) is not easy and susceptible to large errors. The threshold power is also consistent with the observation that no trapping is seen for input powers below about $150 \mathrm{~mW}$.

To verify that these values of trap frequencies are reasonable, we have also measured the trap stiffness using one of the standard techniques, namely, by measuring the power spectrum of Brownian fluctuations of the trapped particle. The rolloff frequency of the spectrum $f_{c}$ can be used to determine the trap stiffness as [10]

$$
k_{\text {trap }}=6 \pi^{2} \eta d f_{c},
$$

where $\eta=1002 \mu \mathrm{N} \mathrm{s} / \mathrm{m}^{2}$ is the viscosity of the surrounding medium and $d=3 \mu \mathrm{m}$ is the diameter of the bead. The amplitude of the noise spectrum (from a sample of length $24 \mathrm{~s}$ ) is shown in Fig. 4, which shows a rolloff frequency of $43 \mathrm{~Hz}$. This gives a trap stiffness of $7.65 \mu \mathrm{N} / \mathrm{m}$ and a trap-oscillation frequency of $3.6 \mathrm{kHz}$. The corresponding frequency measured by our direct parametric-resonance technique is $3.97 \mathrm{kHz}$. Note that the other method relies on the knowledge of the diameter of the bead and the exact coefficient of viscosity, which in turn depends on its shape, environment, stray charges, etc. Moreover, the rolloff frequency determined from Fig. 4 itself has an error of 7\%. Thus, we estimate an error of $20 \%$ in the determination of $k_{\text {trap }}$ or an error of $10 \%$ in the trap frequency [15]. Hence, the $10 \%$ agreement between the two values appears reasonable. The noise spectrum also gives information about the time-time correlations in the bead position. As shown in the inset in Fig. 4, the spectrum

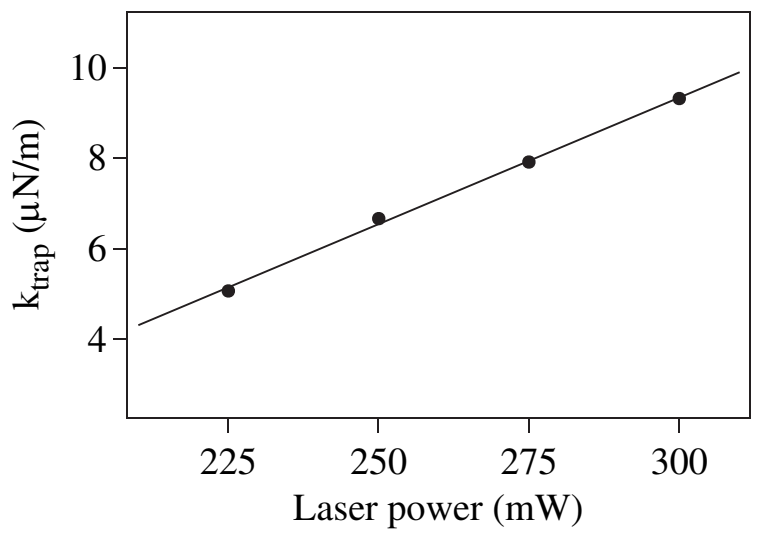

FIG. 3. The trap strength is shown as a function of the input laser power, assuming a bead mass of $1.5 \times 10^{-14} \mathrm{~kg}$. The solid line is a fit to the expected linear behavior. 


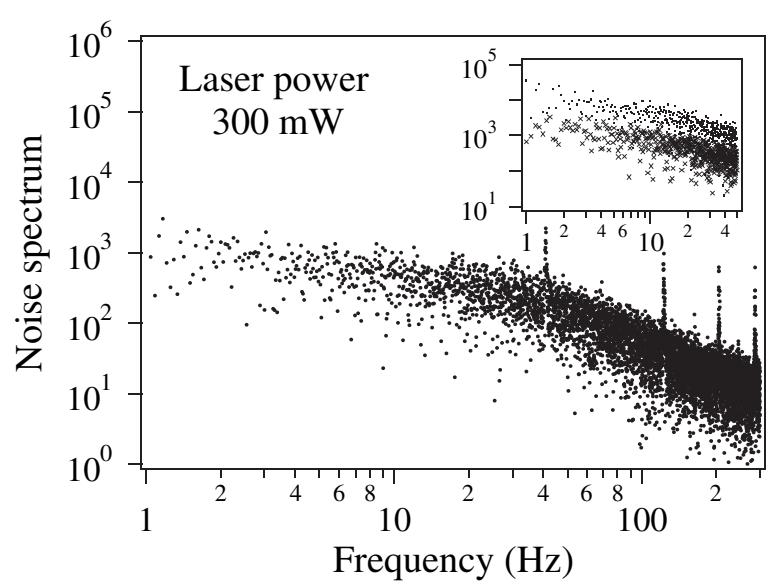

FIG. 4. Fourier spectrum of the Brownian fluctuations of the trapped bead along the $x$ direction. The spectrum (on a log-log scale) shows a knee at a frequency of $43 \mathrm{~Hz}$, which can be related to the trap stiffness as discussed in the text. The inset shows the spectrum with the parametric drive: on resonance (dots) and off resonance (crosses).

with parametric modulation shows a significant increase in low-frequency components on resonance, consistent with the physical picture that reduced damping increases the correlation time.

In summary, we have demonstrated a technique for direct measurement of the oscillation frequency in an optical-tweezers trap. The frequency is measured by parametric excitation of the trapped particle by modulating the trapping power. This is probably the first time that parametric excitation has been studied in a strongly damped system. The signature of resonance is not an increase in the amplitude of oscillation but an increase in the size of Brownian fluctuations; i.e., the energy injected by the parametric drive appears as additional noise. In the experiments reported here, the trapping power was modulated using an AOM. However, most diode lasers and diodepumped lasers used in tweezers experiments allow modulation of the output power by modulating the diode injection current. Thus, it should be possible to do these experiments without the use of an AOM. We have also done experiments where the QPD was replaced with a CCD camera. In these experiments, it was possible to determine the parametric-resonance condition simply by looking at the trapped particle on the video screen: The outline of the bead appeared diffuse and its size increased significantly at the resonance frequency. The resonance is so sharp that, as we change the modulation frequency in steps of $0.2 \mathrm{kHz}$, there is just one frequency (or, at most, two) where the fluctuations increase significantly. It should, therefore, be possible to get a quick and accurate measurement of the trap frequency just by viewing the bead as a function of modulation frequency. The parametric-resonance curve has a width of $1.2 \%$, while the frequency is measured with an uncertainty of only $0.1 \%$. The frequency is consistent with trap strength measured using other techniques. While the trap strength determined using our technique is also "indirect" in the sense that it requires knowledge of the particle mass, the oscillation frequency is measured directly and with high accuracy. This opens up new possibilities in opticaltweezers experiments. For example, the trap frequency depends on parameters such as refractive index of the trapped object, its shape, and its mass. Thus, one might be able to detect changes in these parameters, or sort particles automatically according to their size or shape, by measuring relative changes in the frequency.

We thank Prashant Srinivasan and Vijay Narayan for help with the experiments. This work was supported by the Board of Research in Nuclear Sciences (DAE) and the Life Sciences Research Board (DRDO), Government of India.

*Electronic addresses: vasant@ physics.iisc.ernet.in physics.iisc.ernet.in/ vasant

[1] A. Ashkin, Phys. Rev. Lett. 24, 156 (1970).

[2] A. Ashkin, J. M. Dziedzic, J. E. Bjorkholm, and S. Chu, Opt. Lett. 11, 288 (1986).

[3] T. L. Gustavson et al., Phys. Rev. Lett. 88, 020401 (2002).

[4] A. Ashkin and J. M. Dzeidzic, Science 235, 1517 (1987).

[5] A. Ashkin, J.M. Dzeidzic, and T. Yamane, Nature (London) 330, 769 (1987).

[6] A. Ashkin et al., Nature (London) 348, 346 (1990).

[7] K. Svoboda and S.M. Block, Annu. Rev. Biophys. Biomol. Struct. 23, 247 (1994).

[8] S. B. Smith, Y. Cui, and C. Bustamante, Science 271, 795 (1996).

[9] S. M. Block, L. S. B. Goldstein, and B. J. Schnapp, Nature (London) 348, 348 (1990).

[10] K. Svoboda and S. M. Block, Cell 77, 773 (1994).

[11] L. D. Landau and E. M. Lifshitz, in Mechanics, Course of Theroretical Physics Vol. 1 (Pergamon, Oxford, England, 1976), 3rd ed., Chap. V, p. 80.

[12] V. Natarajan, F. DiFilippo, and D. E. Pritchard, Phys. Rev. Lett. 74, 2855 (1995).

[13] Note that this is contrary to what usually happens in a driven harmonic oscillator: The presence of friction broadens the resonance curve.

[14] R. K. Pathria, Statistical Mechanics (ButterworthHeinemann, Oxford, England, 1996), 2nd ed., Chap. 14, p. 481.

[15] The error in the rolloff technique can be reduced below $10 \%$ by careful experimental control, but we have not attempted this here since we just wanted to show consistency of the value with our technique. 